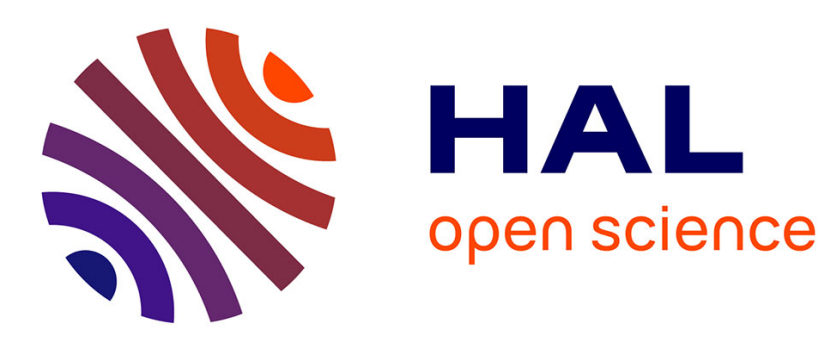

\title{
Adaptive wavelet estimator for a function and its derivatives in an indirect convolution model
}

Christophe Chesneau

\section{To cite this version:}

Christophe Chesneau. Adaptive wavelet estimator for a function and its derivatives in an indirect convolution model. 2012. hal-00508811v2

HAL Id: hal-00508811

https://hal.science/hal-00508811v2

Preprint submitted on 7 Jul 2012

HAL is a multi-disciplinary open access archive for the deposit and dissemination of scientific research documents, whether they are published or not. The documents may come from teaching and research institutions in France or abroad, or from public or private research centers.
L'archive ouverte pluridisciplinaire HAL, est destinée au dépôt et à la diffusion de documents scientifiques de niveau recherche, publiés ou non, émanant des établissements d'enseignement et de recherche français ou étrangers, des laboratoires publics ou privés. 


\title{
Adaptive wavelet estimator for a function and its derivatives in an indirect convolution model
}

\section{Christophe Chesneau}

Received:

\begin{abstract}
We consider an indirect convolution model where $m$ blurred and noise-perturbed functions $f_{1}, \ldots, f_{m}$ are randomly observed. For a fixed $\omega \in$ $\{1, \ldots, m\}$, we want to estimate $f_{\omega}$ and its derivatives. An adaptive nonlinear wavelet estimator using a singular value decomposition is developed. Taking the mean integrated squared error over Besov balls, we prove that it attains a fast rate of convergence.
\end{abstract}

Keywords Deconvolution · Function estimation - Rate of convergence · Wavelets · Hard thresholding.

2000 Mathematics Subject Classification 62G07, 62G20.

\section{Motivations}

We observe $n$ stochastic processes $Y_{1}(t), \ldots, Y_{n}(t), t \in[0,1]$, such that, for any $v \in\{1, \ldots, n\}$,

$$
d Y_{v}(t)=\left(f_{X_{v}} \star g\right)(t) d t+d W_{v}(t)
$$

where

$$
\left(f_{X_{v}} \star g\right)(t)=\int_{0}^{1} f_{X_{v}}(t-u) g(u) d u
$$

$W_{1}(t), \ldots, W_{n}(t)$ are $n$ unobserved independent standard Brownian motions, $g:[0,1] \rightarrow \mathbb{R}$ is a known 1-periodic function, $X_{1}, \ldots, X_{n}$ are $n$ unobserved independent discrete random variables such that, for any $v \in\{1, \ldots, n\}$, the distribution of $X_{v}$ is known, the set of possible values of $X_{v}$ is

$$
X_{v}(\Omega)=\{1, \ldots, m\}, \quad m \in \mathbb{N}^{*},
$$

Laboratoire de Mathématiques Nicolas Oresme, Université de Caen Basse-Normandie, Campus II, Science 3, 14032 Caen, France. E-mail: chesneau@math.unicaen.fr 
and, for any $d \in\{1, \ldots, m\}, f_{d}:[0,1] \rightarrow \mathbb{R}$ is an unknown 1-periodic function. We suppose that $X_{1}, \ldots, X_{n}, W_{1}(t), \ldots, W_{n}(t)$ are independent. For a fixed $\omega \in\{1, \ldots, m\}$, we want to estimate $f_{\omega}$ and its $q$-th derivative $f_{\omega}^{(q)}$ from $Y_{1}(t), \ldots, Y_{n}(t)$. An application of this estimation problem is the following: $m$ blurred and noise-perturbed signals $f_{1}, \ldots, f_{m}$ are randomly observed, and only $f_{\omega}$ is of interest. The blurring process is achieved through the convolution operator $T(h)=(h \star g)(t)=\int_{0}^{1} h(t-u) g(u) d u$.

Remark that, when $X_{1}, \ldots, X_{n}$ are constant random variables with $X_{1}=$ $\ldots=X_{n}=1,(1)$ gives

$$
d \widetilde{Y}(t)=\left(f_{1} \star g\right)(t) d t+\frac{1}{\sqrt{n}} d \widetilde{W}(t),
$$

where

$$
\widetilde{Y}(t)=\frac{1}{n} \sum_{v=1}^{n} Y_{v}(t), \quad \widetilde{W}(t)=\frac{1}{\sqrt{n}} \sum_{v=1}^{n} W_{v}(t) .
$$

Since $\widetilde{W}(t)$ is a standard Brownian motion, (2) becomes the standard convolution model. In this case, the estimation of $f_{1}$ has received a lot of attention. See e.g. Cavalier and Tsybakov (2002), Johnstone et al. (2004), Donoho and Raimondo (2004), Kerkyacharian et al. (2007) and Chesneau (2008).

However, to the best of our knowledge, the estimation of $f_{\omega}$ (and $f_{\omega}^{(q)}$ ) from the general model (1) has not been addressed earlier. Note that the estimation of $f_{\omega}^{(q)}$ is of interest to detect possible bumps, concavity or convexity properties of $f_{\omega}$. In the literature on nonparametric functional estimation, the problem of estimating the derivatives have been investigated by several authors starting with Bhattacharya (1967). For references using wavelet methods, see e.g. Prakasa Rao (1996), Chaubey and Doosti (2005), Chaubey et al. (2006, 2008) and Chesneau (2010).

Considering the ordinary smooth case on $g$ (see (4)), we estimate $f_{\omega}^{(q)}$ (including $f_{\omega}^{(0)}=f_{\omega}$ ) by two wavelet estimators: a linear nonadaptive and a nonlinear adaptive based on the hard thresholding rule. Our adaptive estimator uses a singular value decomposition (SVD), some algebraic tools on the distributions of $X_{1}, \ldots, X_{n}$ and a new version of the "observations thresholding" introduced by Delyon and Juditsky (1996). To evaluate their performances, we adopt the mean integrated squared error (MISE) over Besov balls. Under mild assumptions on the distributions of $X_{1}, \ldots, X_{n}$, we prove that our hard thresholding estimator attains a sharp rate of convergence, close to the one attained by our linear wavelet estimator.

The paper is organized as follows. Assumptions on (1) and some notations are introduced in Section 2. Section 3 briefly describes the wavelet basis and the Besov balls. The estimators are presented in Section 4. The results are set in Section 5. Technical proofs are given in Section 6. 


\section{Assumptions and notations}

Assumptions on $f_{1}, \ldots, f_{m}$ and $g$.

- We assume that $f_{1}, \ldots, f_{m}$ and $g$ belong to $\mathbb{L}_{\text {per }}^{2}([0,1])$ defined by

$$
\mathbb{L}_{\text {per }}^{2}([0,1])=\left\{h ; h \text { is } 1 \text {-periodic and }\left(\int_{0}^{1}|h(x)|^{2} d x\right)^{1 / 2}<\infty\right\} .
$$

- We suppose that there exists a known constant $C_{*}>0$ such that

$$
\sup _{d \in\{1, \ldots, m\}} \sup _{x \in[0,1]}\left|f_{d}^{(q)}(x)\right| \leq C_{*} .
$$

Assumptions on the smoothness of $g$. Any function $h \in \mathbb{L}_{\text {per }}^{2}([0,1])$ can be represented by its Fourier series

$$
h(t)=\sum_{\ell \in \mathbb{Z}} \mathcal{F}_{\ell}(h) e^{2 i \pi \ell t}, \quad t \in[0,1]
$$

where the equality is intended in mean-square convergence sense, and $\mathcal{F}_{\ell}(h)$ denotes the Fourier coefficient given by

$$
\mathcal{F}_{\ell}(h)=\int_{0}^{1} h(x) e^{-2 i \pi \ell x} d x, \quad \ell \in \mathbb{Z}
$$

whenever this integral exists. The notation ${ }^{-}$will be used for the complex conjugate.

We consider the ordinary smooth case on $g$ : there exist three constants, $c_{g}>0, C_{g}>0$ and $\delta>1$, such that, for any $\ell \in \mathbb{Z}, \mathcal{F}_{\ell}(g)$ satisfies

$$
\frac{c_{g}}{\left(1+\ell^{2}\right)^{\delta / 2}} \leq\left|\mathcal{F}_{\ell}(g)\right| \leq \frac{C_{g}}{\left(1+\ell^{2}\right)^{\delta / 2}}
$$

This assumption controls the decay of the Fourier coefficients of $g$, and thus the smoothness of $g$. It is a standard hypothesis usually adopted in the field of nonparametric estimation for deconvolution problems. See e.g. Pensky and Vidakovic (1999), Fan and Koo (2002) and Johnstone et al. (2004).

Assumptions on $X_{1}, \ldots, X_{n}$. Recall that $X_{1}, \ldots, X_{n}$ are unobserved and, for any $v \in\{1, \ldots, n\}$, we let

$$
w_{d}(v)=\mathbb{P}\left(X_{v}=d\right), \quad d \in\{1, \ldots, m\} .
$$

We suppose that the matrix

$$
\Gamma_{n}=\left(\frac{1}{n} \sum_{v=1}^{n} w_{k}(v) w_{\ell}(v)\right)_{(k, \ell) \in\{1, \ldots, m\}^{2}}
$$


satisfies $\operatorname{det}\left(\Gamma_{n}\right)>0$. For the considered $\omega$ (the one which refers to the estimation of $f_{\omega}^{(q)}$ ) and any $v \in\{1, \ldots, n\}$, we set

$$
a_{\omega}(v)=\frac{1}{\operatorname{det}\left(\Gamma_{n}\right)} \sum_{k=1}^{m}(-1)^{k+\omega} \gamma_{\omega, k}^{n} w_{k}(v),
$$

where $\gamma_{\omega, k}^{n}$ denotes the determinant of the minor $(\omega, k)$ of the matrix $\Gamma_{n}$. Then $\left(a_{\omega}(v)\right)_{v \in\{1, \ldots, n\}}$ satisfy

$$
\left(a_{\omega}(1), \ldots, a_{\omega}(n)\right)=\underset{\left(b_{1}, \ldots, b_{n}\right) \in \cap_{d=1}^{m} \mathcal{U}_{\omega, d}}{\operatorname{argmin}} \frac{1}{n} \sum_{v=1}^{n} b_{v}^{2},
$$

where

$$
\mathcal{U}_{\omega, d}=\left\{\left(b_{1}, \ldots, b_{n}\right) \in \mathbb{R}^{n} ; \frac{1}{n} \sum_{v=1}^{n} b_{v} w_{d}(v)=\delta_{\omega, d}\right\}
$$

and $\delta_{\omega, d}$ is the Kronecker delta.

Technical details can be found in Maiboroda (1996).

We set

$$
\rho_{n}=\frac{1}{n} \sum_{v=1}^{n} a_{\omega}^{2}(v)
$$

and we suppose that $\lim _{n \rightarrow \infty} \rho_{n} / n=\infty$.

The sequence $\left(a_{\omega}(v)\right)_{v \in\{1, \ldots, n\}}$ is usually used in the field of nonparametric statistics for mixture density estimation problems. See e.g. Maiboroda (1996), Pokhyl'ko (2005) and Prakasa Rao (2010).

\section{Wavelets and Besov balls}

Periodized Meyer wavelets. We consider an orthonormal wavelet basis generated by dilations and translations of a "father" Meyer-type wavelet $\phi$ and a "mother" Meyer-type wavelet $\psi$. The main features of such wavelets are:

1. the Fourier transforms of $\phi$ and $\psi$ have compact supports with

$$
\left\{\begin{array}{l}
\operatorname{supp}(\mathcal{F}(\phi)) \subset\left[-\frac{4 \pi}{3}, \frac{4 \pi}{3}\right], \\
\operatorname{supp}(\mathcal{F}(\psi)) \subset\left[-\frac{8 \pi}{3},-\frac{2 \pi}{3}\right] \cup\left[\frac{2 \pi}{3}, \frac{8 \pi}{3}\right],
\end{array}\right.
$$

2. the functions $\phi$ and $\psi$ are $\mathcal{C}^{\infty}$. 
For the purpose of this paper, we use the periodized Meyer wavelet bases on the unit interval. For any $x \in[0,1]$, any integer $j$ and any $k \in\left\{0, \ldots, 2^{j}-\right.$ $1\}$, let

$$
\phi_{j, k}(x)=2^{j / 2} \phi\left(2^{j} x-k\right), \quad \psi_{j, k}(x)=2^{j / 2} \psi\left(2^{j} x-k\right)
$$

be the elements of the wavelet basis, and

$$
\phi_{j, k}^{\text {per }}(x)=\sum_{l \in \mathbb{Z}} \phi_{j, k}(x-l), \quad \psi_{j, k}^{\text {per }}(x)=\sum_{l \in \mathbb{Z}} \psi_{j, k}(x-l),
$$

be their periodized versions. There exists an integer $j_{*}$ such that, for any integer $j_{c} \geq j_{*}$, the collection $\mathcal{B}=\left\{\phi_{j_{c}, k}^{\text {per }}, k \in\left\{0, \ldots, 2^{j_{c}}-1\right\} ; \psi_{j, k}^{\text {per }}, \quad j \in\right.$ $\left.\mathbb{N}-\left\{0, \ldots, j_{c}-1\right\}, k \in\left\{0, \ldots, 2^{j}-1\right\}\right\}$ forms an orthonormal basis of $\mathbb{L}_{\text {per }}^{2}([0,1])$. In what follows, the superscript "per" will be dropped to lighten the notation.

Let $j_{c}$ be an integer such that $j_{c} \geq j_{*}$. A function $h \in \mathbb{L}_{\text {per }}^{2}([0,1])$ can be expanded into a wavelet series as

$$
h(x)=\sum_{k=0}^{2^{j_{c}}-1} \alpha_{j_{c}, k} \phi_{j_{c}, k}(x)+\sum_{j=j_{c}}^{\infty} \sum_{k=0}^{2^{j}-1} \beta_{j, k} \psi_{j, k}(x), \quad x \in[0,1],
$$

where

$$
\alpha_{j, k}=\int_{0}^{1} h(x) \bar{\phi}_{j, k}(x) d x, \quad \beta_{j, k}=\int_{0}^{1} h(x) \bar{\psi}_{j, k}(x) d x .
$$

See (Meyer 1992, Vol. 1 Chapter III.11) for a detailed account on periodized orthonormal wavelet bases.

Besov balls. Let $M>0, s>0, p \geq 1$ and $r \geq 1$. A function $h$ belongs to $B_{p, r}^{s}(M)$ if and only if there exists a constant $M_{*}>0$ such that the wavelet coefficients (8) satisfy

$$
\begin{aligned}
& 2^{j_{*}(1 / 2-1 / p)}\left(\sum_{k=0}^{2^{j *}-1}\left|\alpha_{j_{*}, k}\right|^{p}\right)^{1 / p}+\left(\sum_{j=j_{*}}^{\infty}\left(2^{j(s+1 / 2-1 / p)}\left(\sum_{k=0}^{2^{j}-1}\left|\beta_{j, k}\right|^{p}\right)^{1 / p}\right)^{r}\right)^{1 / r} \\
& \leq M_{*} .
\end{aligned}
$$

For a particular choice of parameters $s, p$ and $r$, these sets contain the Hölder and Sobolev balls. See Meyer (1992).

\section{Estimators}

Estimators for wavelet coefficients. The first step to estimate $f_{\omega}^{(q)}$ consists in expanding $f_{\omega}^{(q)}$ on $\mathcal{B}$ and estimating its unknown wavelet coefficients.

For any integer $j \geq j_{*}$ and any $k \in\left\{0, \ldots, 2^{j}-1\right\}$, 
- we estimate $\alpha_{j, k}=\int_{0}^{1} f_{\omega}^{(q)}(x) \bar{\phi}_{j, k}(x) d x$ by

$$
\widehat{\alpha}_{j, k}=\frac{1}{n} \sum_{v=1}^{n} a_{\omega}(v) \sum_{\ell \in \mathcal{C}_{j}}(2 i \pi \ell)^{q} \frac{\overline{\mathcal{F}_{\ell}\left(\phi_{j, k}\right)}}{\mathcal{F}_{\ell}(g)} \int_{0}^{1} e^{-2 i \pi \ell t} d Y_{v}(t),
$$

where $\mathcal{C}_{j}=\operatorname{supp}\left(\mathcal{F}\left(\phi_{j, 0}\right)\right)=\operatorname{supp}\left(\mathcal{F}\left(\phi_{j, k}\right)\right)$ and $a_{\omega}(v)$ is defined by (5),

- we estimate $\beta_{j, k}=\int_{0}^{1} f_{\omega}^{(q)}(x) \bar{\psi}_{j, k}(x) d x$ by

$$
\widehat{\beta}_{j, k}=\frac{1}{n} \sum_{v=1}^{n} Q_{v} \mathbf{1}_{\left\{\left|Q_{v}\right| \leq \eta_{j}\right\}},
$$

where

$$
Q_{v}=a_{\omega}(v) \sum_{\ell \in \mathcal{D}_{j}}(2 i \pi \ell)^{q} \frac{\overline{\mathcal{F}_{\ell}\left(\psi_{j, k}\right)}}{\mathcal{F}_{\ell}(g)} \int_{0}^{1} e^{-2 i \pi \ell t} d Y_{v}(t)
$$

$\mathcal{D}_{j}=\operatorname{supp}\left(\mathcal{F}\left(\psi_{j, 0}\right)\right)=\operatorname{supp}\left(\mathcal{F}\left(\psi_{j, k}\right)\right)$, for any random event $\mathcal{A}, \mathbf{1}_{\mathcal{A}}$ is the indicator function on $\mathcal{A}, a_{\omega}(v)$ is defined by (5),

$$
\eta_{j}=\theta 2^{(\delta+q) j} \sqrt{\frac{n \rho_{n}}{\ln \left(n / \rho_{n}\right)}},
$$

$\rho_{n}$ is defined by $(7)$ and

$$
\theta=\sqrt{3 C_{*}^{2}+2^{\delta} \frac{2(2 \pi)^{2 q}}{c_{g}^{2}}\left(\frac{8 \pi}{3}\right)^{2(\delta+q)}} .
$$

$\left(C_{*}, c_{g}\right.$ and $\delta$ are those in $(3)$ and $\left.(4)\right)$.

Statistical properties of $\widehat{\alpha}_{j, k}$ and $\widehat{\beta}_{j, k}$ are investigated in Propositions 1,3 , 4 and 5.

\section{Remarks.}

- The constructions of $\widehat{\alpha}_{j, k}$ and $\widehat{\beta}_{j, k}$ are based on a SVD technique (see Proposition 1).

- The idea of the thresholding in (10) is to operate a selection on the observations: when, for $v \in\{1, \ldots, n\}, Q_{v}$ is too large, $Y_{v}(t)$ is neglected. From a technical point of view, this allows us to estimate $\beta_{j, k}$ in an optimal way under mild assumptions on $\left(a_{\omega}(v)\right)_{v \in\{1, \ldots, n\}}$ and, a fortiori, on the distributions of $X_{1}, \ldots, X_{n}$. Such a thresholding method has been introduced by Delyon and Juditsky (1996) for regression wavelet estimation. Note that, in the simplest case where $X_{1}, \ldots, X_{n}$ are constant random variables, such a selection is not necessary.

We consider two wavelet estimators for $f_{\omega}^{(q)}$ : a linear estimator and a hard thresholding estimator. 
Linear estimator. Assuming that $f_{\omega}^{(q)} \in B_{p, r}^{s}(M)$ with $p \geq 2$, we define the linear estimator $\widehat{f}_{L}^{(q)}$ by

$$
\widehat{f}_{L}^{(q)}(x)=\sum_{k=0}^{2^{j_{0}}-1} \widehat{\alpha}_{j_{0}, k} \phi_{j_{0}, k}(x)
$$

where $\widehat{\alpha}_{j, k}$ is defined by (9), $j_{0}$ is the integer satisfying

$$
\frac{1}{2}\left(\frac{n}{\rho_{n}}\right)^{1 /(2 s+2 \delta+2 q+1)}<2^{j_{0}} \leq\left(\frac{n}{\rho_{n}}\right)^{1 /(2 s+2 \delta+2 q+1)},
$$

$\rho_{n}$ is defined by (7) and $\delta$ is the one in (4).

Note that $\widehat{f}_{L}^{(q)}$ is not adaptive since it depends on $s$.

Hard thresholding estimator. We define the hard thresholding estimator $\widehat{f}_{H}^{(q)}$ by

$$
\widehat{f}_{H}^{(q)}(x)=\sum_{k=0}^{2^{j_{*}}-1} \widehat{\alpha}_{j_{*}, k} \phi_{j_{*}, k}(x)+\sum_{j=j_{*}}^{j_{1}} \sum_{k=0}^{2^{j}-1} \widehat{\beta}_{j, k} \mathbf{1}_{\left\{\left|\widehat{\beta}_{j, k}\right| \geq \kappa \lambda_{j}\right\}} \psi_{j, k}(x),
$$

where $\widehat{\alpha}_{j_{*}, k}$ is defined by (9), $\widehat{\beta}_{j, k}$ by $(10), j_{1}$ is the integer satisfying

$$
\frac{1}{2}\left(\frac{n}{\rho_{n}}\right)^{1 /(2 \delta+2 q+1)}<2^{j_{1}} \leq\left(\frac{n}{\rho_{n}}\right)^{1 /(2 \delta+2 q+1)}
$$

$\kappa \geq 8 / 3+2+2 \sqrt{16 / 9+4}, \lambda_{j}$ is the threshold

$$
\lambda_{j}=\theta 2^{(\delta+q) j} \sqrt{\frac{\rho_{n} \ln \left(n / \rho_{n}\right)}{n}},
$$

$\rho_{n}$ is defined by (7), $\theta$ by (12) and $\delta$ is the one in (4).

Note that $\widehat{f}_{H}^{(q)}$ is adaptive.

The feature of the hard thresholding estimator is to only estimate the "large" unknown wavelet coefficients of $f_{\omega}^{(q)}$ which contain the main characteristics of $f_{\omega}^{(q)}$.

Hard thresholding estimators for other deconvolution problems than (1) can be found in Fan and Koo (2002), Johnstone et al. (2004), Willer (2005) and Cavalier and Raimondo (2007).

\section{Results}

Upper bounds for $\widehat{f}_{L}^{(q)}$ and $\widehat{f}_{H}^{(q)}$ are given in Theorems 1 and 2 below. Further details on our statistical approach can be found in Tsybakov (2004). 
Theorem 1 Consider (1) under the assumptions of Section 2. Suppose that $f_{\omega}^{(q)} \in B_{p, r}^{s}(M)$ with $s>0, p \geq 2$ and $r \geq 1$. Let $\widehat{f}_{L}^{(q)}$ be (13). Then there exists a constant $C>0$ such that

$$
\mathbb{E}\left(\int_{0}^{1}\left(\widehat{f}_{L}^{(q)}(x)-f_{\omega}^{(q)}(x)\right)^{2} d x\right) \leq C\left(\frac{\rho_{n}}{n}\right)^{2 s /(2 s+2 \delta+2 q+1)} .
$$

The proof of Theorem 1 uses a moment inequality on (9) and a suitable decomposition of the MISE. Note that $\widehat{f}_{L}^{(q)}$ is constructed to minimize the MISE as much as possible. For this reason, our benchmark will be the rate of convergence $\left(\rho_{n} / n\right)^{2 s /(2 s+2 \delta+2 q+1)}$.

Theorem 2 Consider (1) under the assumptions of Section 2. Let $\widehat{f}_{H}^{(q)}$ be (14). Suppose that $f_{\omega}^{(q)} \in B_{p, r}^{s}(M)$ with $r \geq 1,\{p \geq 2$ and $s>0\}$ or $\{p \in[1,2)$ and $s>(2 \delta+2 q+1) / p\}$. Then there exists a constant $C>0$ such that

$$
\mathbb{E}\left(\int_{0}^{1}\left(\widehat{f}_{H}^{(q)}(x)-f_{\omega}^{(q)}(x)\right)^{2} d x\right) \leq C\left(\frac{\rho_{n} \ln \left(n / \rho_{n}\right)}{n}\right)^{2 s /(2 s+2 \delta+2 q+1)} .
$$

The proof of Theorem 2 is based on several probability results (moment inequalities, concentration inequality,...) and a suitable decomposition of the MISE.

Theorem 2 shows that, besides being adaptive, $\widehat{f}_{H}^{(q)}$ attains the same rate of convergence than the one of $\widehat{f}_{L}^{(q)}$ up to a logarithmic term. Naturally, in the simplest case where $X_{1}, \ldots, X_{n}$ are constant random variables with $X_{1}=\ldots=X_{n}=1$ (so $\rho_{n}=1$ and $f_{\omega}^{(q)}=f_{1}^{(q)}$ ), the rate of convergence attained by $\widehat{f}_{H}^{(q)}$ becomes the standard one for $(2)$ i.e. $(\ln n / n)^{2 s /(2 s+2 \delta+2 q+1)}$. See Johnstone et al. (2004) and Chesneau (2010).

Conclusion and perspectives. Considering (1), we have developed a new adaptive estimator $\widehat{f}_{H}^{(q)}$ for $f_{\omega}^{(q)}$. It is based on a SVD, wavelets and the hard thresholding rule. It attains a sharp rate of convergence for a wide class of functions. Possible perspectives of this work are

- to investigate the case where the distributions of $X_{1}, \ldots, X_{n}$ are unknown,

- to potentially improve the estimation of $f_{\omega}^{(q)}$ by considering other kinds of thresholding rules as the block thresholding one (BlockJS, ...). See e.g. Cai (1999, 2002), Pensky and Sapatinas (2009) and Petsa and Sapatinas (2009).

All these aspects need further investigations that we leave for a future work.

\section{Proofs}

In this section, $C$ represents a positive constant which may differ from one term to another. 
6.1 Auxiliary results

Proposition 1 For any integer $j \geq j_{*}$ and any $k \in\left\{0, \ldots, 2^{j}-1\right\}$, let $\alpha_{j, k}$ and $\beta_{j, k}$ be the wavelet coefficients (8) of $f_{\omega}^{(q)}$. Then

- $\widehat{\alpha}_{j, k}$ defined by (9) is an unbiased estimator of $\alpha_{j, k}$,

- for $\left(Q_{v}\right)_{v \in\{1, \ldots, n\}}$ defined by (11), we have

$$
\mathbb{E}\left(\frac{1}{n} \sum_{v=1}^{n} Q_{v}\right)=\beta_{j, k}
$$

Proof of Proposition 1. We have

$$
\int_{0}^{1} e^{-2 i \pi \ell t} d Y_{v}(t)=\int_{0}^{1} e^{-2 i \pi \ell t}\left(f_{X_{v}} \star g\right)(t) d t+\int_{0}^{1} e^{-2 i \pi \ell t} d W_{v}(t)
$$

with

$$
\int_{0}^{1} e^{-2 i \pi \ell t}\left(f_{X_{v}} \star g\right)(t) d t=\mathcal{F}_{\ell}\left(f_{X_{v}} \star g\right)=\mathcal{F}_{\ell}\left(f_{X_{v}}\right) \mathcal{F}_{\ell}(g) .
$$

Since $\mathbb{E}\left(\int_{0}^{1} e^{-2 i \pi \ell t} d W_{v}(t)\right)=0$, we have

$$
\begin{aligned}
\mathbb{E}\left(\int_{0}^{1} e^{-2 i \pi \ell t} d Y_{v}(t)\right) & =\mathbb{E}\left(\mathcal{F}_{\ell}\left(f_{X_{v}}\right)\right) \mathcal{F}_{\ell}(g)+\mathbb{E}\left(\int_{0}^{1} e^{-2 i \pi \ell t} d W_{v}(t)\right) \\
& =\sum_{d=1}^{m} w_{d}(v) \mathcal{F}_{\ell}\left(f_{d}\right) \mathcal{F}_{\ell}(g) .
\end{aligned}
$$

Note that, since $f_{\omega}$ is 1-periodic, for any $u \in\{0, \ldots, q\}, f_{\omega}^{(u)}$ is 1-periodic and $f_{\omega}^{(u)}(0)=f_{\omega}^{(u)}(1)$. By $q$ integrations by parts, for any $\ell \in \mathbb{Z}$, we obtain

$$
(2 i \pi \ell)^{q} \mathcal{F}_{\ell}\left(f_{\omega}\right)=\mathcal{F}_{\ell}\left(f_{\omega}^{(q)}\right) .
$$

It follows from (17), (6), (18) and the Parseval-Plancherel theorem that

$$
\begin{aligned}
\mathbb{E}\left(\widehat{\alpha}_{j, k}\right) & =\frac{1}{n} \sum_{v=1}^{n} a_{\omega}(v) \sum_{\ell \in \mathcal{C}_{j}}(2 i \pi \ell)^{q} \frac{\overline{\mathcal{F}_{\ell}\left(\phi_{j, k}\right)}}{\mathcal{F}_{\ell}(g)} \mathbb{E}\left(\int_{0}^{1} e^{-2 i \pi \ell t} d Y_{v}(t)\right) \\
& =\frac{1}{n} \sum_{v=1}^{n} a_{\omega}(v) \sum_{\ell \in \mathcal{C}_{j}}(2 i \pi \ell)^{q} \frac{\overline{\mathcal{F}_{\ell}\left(\phi_{j, k}\right)}}{\mathcal{F}_{\ell}(g)} \sum_{d=1}^{m} w_{d}(v) \mathcal{F}_{\ell}\left(f_{d}\right) \mathcal{F}_{\ell}(g) \\
& =\sum_{d=1}^{m}\left(\sum_{\ell \in \mathcal{C}_{j}}(2 i \pi \ell)^{q} \overline{\mathcal{F}_{\ell}\left(\phi_{j, k}\right)} \mathcal{F}_{\ell}\left(f_{d}\right)\right) \frac{1}{n} \sum_{v=1}^{n} a_{\omega}(v) w_{d}(v) \\
& =\sum_{\ell \in \mathcal{C}_{j}} \frac{\mathcal{F}_{\ell}\left(\phi_{j, k}\right)}{}(2 i \pi \ell)^{q} \mathcal{F}_{\ell}\left(f_{\omega}\right)=\sum_{\ell \in \mathcal{C}_{j}} \frac{\mathcal{F}_{\ell}\left(\phi_{j, k}\right)}{\mathcal{F}_{\ell}}\left(f_{\omega}^{(q)}\right) \\
& =\int_{0}^{1} \bar{\phi}_{j, k}(x) f_{\omega}^{(q)}(x) d x=\alpha_{j, k} .
\end{aligned}
$$


Similarly, taking $\psi$ instead of $\phi$, we prove that $\mathbb{E}\left(\frac{1}{n} \sum_{v=1}^{n} Q_{v}\right)=\beta_{j, k}$.

This completes the proof of Proposition 1.

Proposition 2 For any integer $j \geq j_{*}$ and any $k \in\left\{0, \ldots, 2^{j}-1\right\}$, set

$$
R_{j, k}=\sum_{\ell \in \mathcal{C}_{j}}(2 i \pi \ell)^{q} \frac{\overline{\mathcal{F}_{\ell}\left(\phi_{j, k}\right)}}{\mathcal{F}_{\ell}(g)} \int_{0}^{1} e^{-2 i \pi \ell t} d Y_{v}(t)
$$

Then

$$
\mathbb{E}\left(\left|R_{j, k}\right|^{2}\right) \leq \theta^{2} 2^{2(\delta+q) j},
$$

where $\theta$ is defined by (12).

Proof of Proposition 2. We have

$$
\mathbb{E}\left(\left|R_{j, k}\right|^{2}\right)=\mathbb{E}\left(\mathbb{E}\left(\left|R_{j, k}\right|^{2} \mid X_{v}\right)\right)=\mathbb{E}\left(\mathbb{V}\left(R_{j, k} \mid X_{v}\right)\right)+\mathbb{E}\left(\left|\mathbb{E}\left(R_{j, k} \mid X_{v}\right)\right|^{2}\right) .
$$

Let us bound $\mathbb{E}\left(\mathbb{V}\left(R_{j, k} \mid X_{v}\right)\right)$ and $\mathbb{E}\left(\left|\mathbb{E}\left(R_{j, k} \mid X_{v}\right)\right|^{2}\right)$ in turn.

Upper bound for $\mathbb{E}\left(\mathbb{V}\left(R_{j, k} \mid X_{v}\right)\right)$. Since $\left(e^{-2 i \pi \ell t}\right)_{\ell \in \mathbb{Z}}$ is an orthonormal basis of $\mathbb{L}_{\text {per }}^{2}([0,1])$ and $X_{1}, \ldots, X_{n}, W_{1}(t), \ldots, W_{n}(t)$ are independent, $\left(\int_{0}^{1} e^{-2 i \pi \ell t} d Y_{v}(t)\right)_{\ell \in \mathcal{C}_{j}}$ conditionally to $X_{v}$ are also independent. Therefore

$$
\mathbb{V}\left(R_{j, k} \mid X_{v}\right)=\sum_{\ell \in \mathcal{C}_{j}}(2 \pi \ell)^{2 q} \frac{\left|\mathcal{F}_{\ell}\left(\phi_{j, k}\right)\right|^{2}}{\left|\mathcal{F}_{\ell}(g)\right|^{2}} \mathbb{V}\left(\int_{0}^{1} e^{-2 i \pi \ell t} d Y_{v}(t) \mid X_{v}\right) .
$$

Using the elementary inequality $(x+y)^{2} \leq 2\left(x^{2}+y^{2}\right),(x, y) \in \mathbb{R}^{2}$, the independence of $X_{1}, \ldots, X_{n}, W_{1}(t), \ldots, W_{n}(t),(16)$ and the fact that $\left(e^{-2 i \pi \ell t}\right)_{\ell \in \mathbb{Z}}$ is an orthonormal basis of $\mathbb{L}_{\text {per }}^{2}([0,1])$, we obtain

$$
\begin{aligned}
& \mathbb{V}\left(\int_{0}^{1} e^{-2 i \pi \ell t} d Y_{v}(t) \mid X_{v}\right) \leq \mathbb{E}\left(\left|\int_{0}^{1} e^{-2 i \pi \ell t} d Y_{v}(t)\right|^{2} \mid X_{v}\right) \\
& \leq 2\left(\left|\int_{0}^{1} e^{-2 i \pi \ell t}\left(f_{X_{v}} \star g\right)(t) d t\right|^{2}+\mathbb{E}\left(\left|\int_{0}^{1} e^{-2 i \pi \ell t} d W_{v}(t)\right|^{2}\right)\right) \\
& =2\left(\left|\mathcal{F}_{\ell}\left(f_{X_{v}}\right)\right|^{2}\left|\mathcal{F}_{\ell}(g)\right|^{2}+1\right) .
\end{aligned}
$$

Hence

$$
\mathbb{E}\left(\mathbb{V}\left(\int_{0}^{1} e^{-2 i \pi \ell t} d Y_{v}(t) \mid X_{v}\right)\right) \leq 2\left(\mathbb{E}\left(\left|\mathcal{F}_{\ell}\left(f_{X_{v}}\right)\right|^{2}\right)\left|\mathcal{F}_{\ell}(g)\right|^{2}+1\right) .
$$

It follows from (20) and (21) that

$$
\mathbb{E}\left(\mathbb{V}\left(R_{j, k} \mid X_{v}\right)\right) \leq 2(A+B),
$$


where

$$
A=\sum_{\ell \in \mathcal{C}_{j}}(2 \pi \ell)^{2 q}\left|\mathcal{F}_{\ell}\left(\phi_{j, k}\right)\right|^{2} \mathbb{E}\left(\left|\mathcal{F}_{\ell}\left(f_{X_{v}}\right)\right|^{2}\right)
$$

and

$$
B=\sum_{\ell \in \mathcal{C}_{j}}(2 \pi \ell)^{2 q} \frac{\left|\mathcal{F}_{\ell}\left(\phi_{j, k}\right)\right|^{2}}{\left|\mathcal{F}_{\ell}(g)\right|^{2}}
$$

Let us now bound $A$ and $B$ in turn.

Upper bound for $A$. It follows from (18) and (3) that

$$
(2 \pi \ell)^{2 q} \mathbb{E}\left(\left|\mathcal{F}_{\ell}\left(f_{X_{v}}\right)\right|^{2}\right)=\mathbb{E}\left(\left|\mathcal{F}_{\ell}\left(f_{X_{v}}^{(q)}\right)\right|^{2}\right) \leq C_{*}^{2}
$$

The Parseval-Plancherel theorem gives $\sum_{\ell \in \mathcal{C}_{j}}\left|\mathcal{F}_{\ell}\left(\phi_{j, k}\right)\right|^{2}=\int_{0}^{1}\left|\phi_{j, k}(x)\right|^{2} d x=$ 1. So

$$
A \leq C_{*}^{2} \sum_{\ell \in \mathcal{C}_{j}}\left|\mathcal{F}_{\ell}\left(\phi_{j, k}\right)\right|^{2}=C_{*}^{2}
$$

Upper bound for $B$. The assumption (4) and the elementary inequality $\mid x+$ $\left.y\right|^{\delta} \leq 2^{\delta-1}\left(|x|^{\delta}+|y|^{\delta}\right),(x, y) \in \mathbb{R}^{2}$, imply that

$$
\begin{aligned}
\sup _{\ell \in \mathcal{C}_{j}} \frac{(2 \pi \ell)^{2 q}}{\left|\mathcal{F}_{\ell}(g)\right|^{2}} & \leq \frac{(2 \pi)^{2 q}}{c_{g}^{2}} \sup _{\ell \in \mathcal{C}_{j}} \ell^{2 q}\left(1+\ell^{2}\right)^{\delta} \leq 2^{\delta-1} \frac{(2 \pi)^{2 q}}{c_{g}^{2}} \sup _{\ell \in \mathcal{C}_{j}} \ell^{2 q}\left(1+\ell^{2 \delta}\right) \\
& \leq 2^{\delta-1} \frac{2(2 \pi)^{2 q}}{c_{g}^{2}}\left(\frac{8 \pi}{3}\right)^{2(\delta+q)} 2^{2(\delta+q) j} .
\end{aligned}
$$

Using again the Parseval-Plancherel theorem, we obtain

$$
\begin{aligned}
B & \leq 2^{\delta-1} \frac{2(2 \pi)^{2 q}}{c_{g}^{2}}\left(\frac{8 \pi}{3}\right)^{2(\delta+q)} 2^{2(\delta+q) j} \sum_{\ell \in \mathcal{C}_{j}}\left|\mathcal{F}_{\ell}\left(\phi_{j, k}\right)\right|^{2} \\
& =2^{\delta-1} \frac{2(2 \pi)^{2 q}}{c_{g}^{2}}\left(\frac{8 \pi}{3}\right)^{2(\delta+q)} 2^{2(\delta+q) j} .
\end{aligned}
$$

Putting (22), (23) and (24) together, we have

$$
\mathbb{E}\left(\mathbb{V}\left(R_{j, k} \mid X_{v}\right)\right) \leq 2\left(C_{*}^{2}+2^{\delta-1} \frac{2(2 \pi)^{2 q}}{c_{g}^{2}}\left(\frac{8 \pi}{3}\right)^{2(\delta+q)}\right) 2^{2(\delta+q) j}
$$

Upper bound for $\mathbb{E}\left(\left|\mathbb{E}\left(R_{j, k} \mid X_{v}\right)\right|^{2}\right)$. We have $\mathbb{E}\left(\int_{0}^{1} e^{-2 i \pi \ell t} d W_{v}(t)\right)=0$ and, by $(16)$,

$$
\mathbb{E}\left(\int_{0}^{1} e^{-2 i \pi \ell t}\left(f_{X_{v}} \star g\right)(t) d t \mid X_{v}\right)=\mathcal{F}_{\ell}\left(f_{X_{v}}\right) \mathcal{F}_{\ell}(g) .
$$


Therefore, using (18), we obtain

$$
\begin{aligned}
\mathbb{E}\left(R_{j, k} \mid X_{v}\right) & =\sum_{\ell \in \mathcal{C}_{j}}(2 i \pi \ell)^{q} \frac{\overline{\mathcal{F}_{\ell}\left(\phi_{j, k}\right)}}{\mathcal{F}_{\ell}(g)} \mathbb{E}\left(\int_{0}^{1} e^{-2 i \pi \ell t} d Y_{v}(t) \mid X_{v}\right) \\
& =\sum_{\ell \in \mathcal{C}_{j}}(2 i \pi \ell)^{q} \frac{\overline{\mathcal{F}_{\ell}\left(\phi_{j, k}\right)}}{\mathcal{F}_{\ell}(g)} \mathcal{F}_{\ell}\left(f_{X_{v}}\right) \mathcal{F}_{\ell}(g) \\
& =\sum_{\ell \in \mathcal{C}_{j}} \overline{\mathcal{F}_{\ell}\left(\phi_{j, k}\right)} \mathcal{F}_{\ell}\left(f_{X_{v}}^{(q)}\right) .
\end{aligned}
$$

The Parseval-Plancherel theorem gives

$$
\mathbb{E}\left(R_{j, k} \mid X_{v}\right)=\int_{0}^{1} \bar{\phi}_{j, k}(x) f_{X_{v}}^{(q)}(x) d x .
$$

It follows from (3) and the Cauchy-Schwarz inequality that

$$
\begin{aligned}
\mathbb{E}\left(\left|\mathbb{E}\left(R_{j, k} \mid X_{v}\right)\right|^{2}\right) & \leq \mathbb{E}\left(\left(\int_{0}^{1}\left|\phi_{j, k}(x)\right|\left|f_{X_{v}}^{(q)}(x)\right| d x\right)^{2}\right) \\
& \leq C_{*}^{2} \int_{0}^{1}\left|\phi_{j, k}(x)\right|^{2} d x=C_{*}^{2} .
\end{aligned}
$$

Putting (19), (25) and (26) together, we obtain

$$
\begin{aligned}
\mathbb{E}\left(\left|R_{j, k}\right|^{2}\right) & \leq 2\left(C_{*}^{2}+2^{\delta-1} \frac{2(2 \pi)^{2 q}}{c_{g}^{2}}\left(\frac{8 \pi}{3}\right)^{2(\delta+q)}\right) 2^{2(\delta+q) j}+C_{*}^{2} \\
& \leq \theta^{2} 2^{2(\delta+q) j} .
\end{aligned}
$$

The proof of Proposition 2 is complete.

Proposition 3 For any integer $j \geq j_{*}$ and any $k \in\left\{0, \ldots, 2^{j}-1\right\}$, let $\alpha_{j, k}$ be the wavelet coefficient (8) of $f_{\omega}^{(q)}$ and $\widehat{\alpha}_{j, k}$ be (9). Then there exists a constant $C>0$ such that

$$
\mathbb{E}\left(\left|\widehat{\alpha}_{j, k}-\alpha_{j, k}\right|^{2}\right) \leq C 2^{2(\delta+q) j} \frac{\rho_{n}}{n} .
$$

Proof of Proposition 3. By Proposition 1 , we have $\mathbb{E}\left(\widehat{\alpha}_{j, k}\right)=\alpha_{j, k}$. Therefore, using the independence of $Y_{1}(t), \ldots, Y_{n}(t)$, we obtain

$$
\begin{aligned}
\mathbb{E}\left(\left|\widehat{\alpha}_{j, k}-\alpha_{j, k}\right|^{2}\right) & =\mathbb{V}\left(\widehat{\alpha}_{j, k}\right) \\
& =\frac{1}{n^{2}} \sum_{v=1}^{n} a_{\omega}^{2}(v) \mathbb{V}\left(\sum_{\ell \in \mathcal{C}_{j}}(2 i \pi \ell)^{q} \frac{\overline{\mathcal{F}_{\ell}\left(\phi_{j, k}\right)}}{\mathcal{F}_{\ell}(g)} \int_{0}^{1} e^{-2 i \pi \ell t} d Y_{v}(t)\right) .
\end{aligned}
$$


Proposition 2 implies that

$$
\begin{aligned}
& \mathbb{V}\left(\sum_{\ell \in \mathcal{C}_{j}}(2 i \pi \ell)^{q} \frac{\overline{\mathcal{F}_{\ell}\left(\phi_{j, k}\right)}}{\mathcal{F}_{\ell}(g)} \int_{0}^{1} e^{-2 i \pi \ell t} d Y_{v}(t)\right) \\
& \leq \mathbb{E}\left(\left|\sum_{\ell \in \mathcal{C}_{j}}(2 i \pi \ell)^{q} \frac{\overline{\mathcal{F}_{\ell}\left(\phi_{j, k}\right)}}{\mathcal{F}_{\ell}(g)} \int_{0}^{1} e^{-2 i \pi \ell t} d Y_{v}(t)\right|^{2}\right) \leq \theta^{2} 2^{2(\delta+q) j} .
\end{aligned}
$$

It follows from (27) and (28) that

$$
\mathbb{E}\left(\left|\widehat{\alpha}_{j, k}-\alpha_{j, k}\right|^{2}\right) \leq \theta^{2} 2^{2(\delta+q) j} \frac{1}{n^{2}} \sum_{v=1}^{n} a_{\omega}^{2}(v)=C 2^{2(\delta+q) j} \frac{\rho_{n}}{n} .
$$

The proof of Proposition 3 is complete.

Proposition 4 For any integer $j \geq j_{*}$ and any $k \in\left\{0, \ldots, 2^{j}-1\right\}$, let $\beta_{j, k}$ be the wavelet coefficient (8) of $f_{\omega}^{(q)}$ and $\widehat{\beta}_{j, k}$ be (10). Then there exists a constant $C>0$ such that

$$
\mathbb{E}\left(\left|\widehat{\beta}_{j, k}-\beta_{j, k}\right|^{4}\right) \leq C 2^{4(\delta+q) j} \frac{\left(\rho_{n} \ln \left(n / \rho_{n}\right)\right)^{2}}{n^{2}} .
$$

Proof of Proposition 4. Thanks to Proposition 1, we have

$$
\beta_{j, k}=\mathbb{E}\left(\frac{1}{n} \sum_{v=1}^{n} Q_{v}\right)=\frac{1}{n} \sum_{v=1}^{n} \mathbb{E}\left(Q_{v} \mathbf{1}_{\left\{\left|Q_{v}\right| \leq \eta_{j}\right\}}\right)+\frac{1}{n} \sum_{v=1}^{n} \mathbb{E}\left(Q_{v} \mathbf{1}_{\left\{\left|Q_{v}\right|>\eta_{j}\right\}}\right)
$$

By the elementary inequality $(x+y)^{4} \leq 8\left(x^{4}+y^{4}\right),(x, y) \in \mathbb{R}^{2}$, we have

$$
\begin{aligned}
& \mathbb{E}\left(\left|\widehat{\beta}_{j, k}-\beta_{j, k}\right|^{4}\right) \\
& =\mathbb{E}\left(\left|\frac{1}{n} \sum_{v=1}^{n}\left(Q_{v} \mathbf{1}_{\left\{\left|Q_{v}\right| \leq \eta_{j}\right\}}-\mathbb{E}\left(Q_{v} \mathbf{1}_{\left\{\left|Q_{v}\right| \leq \eta_{j}\right\}}\right)\right)-\frac{1}{n} \sum_{v=1}^{n} \mathbb{E}\left(Q_{v} \mathbf{1}_{\left\{\left|Q_{v}\right|>\eta_{j}\right\}}\right)\right|^{4}\right) \\
& \leq 8(A+B),
\end{aligned}
$$

where

$$
A=\mathbb{E}\left(\left|\frac{1}{n} \sum_{v=1}^{n}\left(Q_{v} \mathbf{1}_{\left\{\left|Q_{v}\right| \leq \eta_{j}\right\}}-\mathbb{E}\left(Q_{v} \mathbf{1}_{\left\{\left|Q_{v}\right| \leq \eta_{j}\right\}}\right)\right)\right|^{4}\right)
$$

and

$$
B=\left|\frac{1}{n} \sum_{v=1}^{n} \mathbb{E}\left(Q_{v} \mathbf{1}_{\left\{\left|Q_{v}\right|>\eta_{j}\right\}}\right)\right|^{4}
$$

Let us bound $A$ and $B$ in turn.

Upper bound for $A$. We need the Rosenthal inequality presented in lemma below (see Rosenthal (1970)). 
Lemma 1 (Rosenthal's inequality) Let $p \geq 2, n \in \mathbb{N}^{*}$ and $\left(U_{v}\right)_{v \in\{1, \ldots, n\}}$ be $n$ zero mean independent random variables such that, for any $v \in\{1, \ldots, n\}$, $\mathbb{E}\left(\left|U_{v}\right|^{p}\right)<\infty$. Then there exists a constant $C>0$ such that

$$
\mathbb{E}\left(\left|\sum_{v=1}^{n} U_{v}\right|^{p}\right) \leq C \max \left(\sum_{v=1}^{n} \mathbb{E}\left(\left|U_{v}\right|^{p}\right),\left(\sum_{v=1}^{n} \mathbb{E}\left(U_{v}^{2}\right)\right)^{p / 2}\right) .
$$

Set, for any $v \in\{1, \ldots, n\}$,

$$
U_{v}=Q_{v} \mathbf{1}_{\left\{\left|Q_{v}\right| \leq \eta_{j}\right\}}-\mathbb{E}\left(Q_{v} \mathbf{1}_{\left\{\left|Q_{v}\right| \leq \eta_{j}\right\}}\right) .
$$

Then, for any $v \in\{1, \ldots, n\}$, we have $\mathbb{E}\left(U_{v}\right)=0$ and, using Proposition 2 with $\psi$ instead of $\phi$, for any $b \in\{2,4\}$,

$\mathbb{E}\left(\left|U_{v}\right|^{b}\right) \leq 2^{b} \mathbb{E}\left(\left|Q_{v}\right|^{b} \mathbf{1}_{\left\{\left|Q_{v}\right| \leq \eta_{j}\right\}}\right) \leq 2^{b} \eta_{j}^{b-2} \mathbb{E}\left(\left|Q_{v}\right|^{2}\right) \leq 2^{b} \theta^{2} \eta_{j}^{b-2} 2^{2(\delta+q) j} a_{\omega}^{2}(v)$.

It follows from the Rosenthal inequality and $\rho_{n}<n / e$ that

$$
\begin{aligned}
A & =\frac{1}{n^{4}} \mathbb{E}\left(\left|\sum_{v=1}^{n} U_{v}\right|^{4} \leq C \frac{1}{n^{4}} \max \left(\sum_{v=1}^{n} \mathbb{E}\left(\left|U_{v}\right|^{4}\right),\left(\sum_{v=1}^{n} \mathbb{E}\left(\left|U_{v}\right|^{2}\right)\right)^{2}\right)\right. \\
& \leq C \frac{1}{n^{4}} \max \left(2^{4} \theta^{2} \eta_{j}^{2} 2^{2(\delta+q) j} \sum_{v=1}^{n} a_{\omega}^{2}(v),\left(2^{2} \theta^{2} 2^{2(\delta+q) j} \sum_{v=1}^{n} a_{\omega}^{2}(v)\right)^{2}\right) \\
& \leq C \frac{1}{n^{4}} \max \left(2^{4(\delta+q) j} \frac{n^{2} \rho_{n}^{2}}{\ln \left(n / \rho_{n}\right)}, 2^{4(\delta+q) j} n^{2} \rho_{n}^{2}\right)=C 2^{4(\delta+q) j} \frac{\rho_{n}^{2}}{n^{2}} .
\end{aligned}
$$

Upper bound for $B$. Using the inequality: $\mathbf{1}_{\left\{\left|Q_{v}\right|>\eta_{j}\right\}} \leq\left(1 / \eta_{j}\right)\left|Q_{v}\right|$, and again Proposition 2 with $\psi$ instead of $\phi$, we obtain

$$
\begin{aligned}
\frac{1}{n} \sum_{v=1}^{n} \mathbb{E}\left(\left|Q_{v}\right| \mathbf{1}_{\left\{\left|Q_{v}\right|>\eta_{j}\right\}}\right) & \leq \frac{1}{\eta_{j}}\left(\frac{1}{n} \sum_{v=1}^{n} \mathbb{E}\left(\left|Q_{v}\right|^{2}\right)\right) \\
& \leq \frac{1}{\theta 2^{(\delta+q) j}} \sqrt{\frac{\ln \left(n / \rho_{n}\right)}{n \rho_{n}}} \theta^{2} 2^{2(\delta+q) j} \rho_{n} \\
& =\theta 2^{(\delta+q) j} \sqrt{\frac{\rho_{n} \ln \left(n / \rho_{n}\right)}{n}} .
\end{aligned}
$$

Hence

$$
B \leq C 2^{4(\delta+q) j} \frac{\left(\rho_{n} \ln \left(n / \rho_{n}\right)\right)^{2}}{n^{2}} .
$$

It follows from $(30),(31),(33)$ and $\rho_{n}<n / e$ that

$$
\begin{aligned}
\mathbb{E}\left(\left|\widehat{\beta}_{j, k}-\beta_{j, k}\right|^{4}\right) & \leq C\left(2^{4(\delta+q) j} \frac{\rho_{n}^{2}}{n^{2}}+2^{4(\delta+q) j} \frac{\left(\rho_{n} \ln \left(n / \rho_{n}\right)\right)^{2}}{n^{2}}\right) \\
& \leq C 2^{4(\delta+q) j} \frac{\left(\rho_{n} \ln \left(n / \rho_{n}\right)\right)^{2}}{n^{2}} .
\end{aligned}
$$

This completes the proof of Proposition 4. 
Proposition 5 For any integer $j \geq j_{*}$ and any $k \in\left\{0, \ldots, 2^{j}-1\right\}$, let $\beta_{j, k}$ be the wavelet coefficient (8) of $f_{\omega}^{(q)}, \widehat{\beta}_{j, k}$ be (10) and $\lambda_{j}$ be (15). Then, for any $\kappa \geq 8 / 3+2+2 \sqrt{16 / 9+4}$,

$$
\mathbb{P}\left(\left|\widehat{\beta}_{j, k}-\beta_{j, k}\right| \geq \kappa \lambda_{j} / 2\right) \leq 2\left(\frac{\rho_{n}}{n}\right)^{2} .
$$

Proof of Proposition 5. Using (29), we have

$$
\begin{aligned}
& \left|\widehat{\beta}_{j, k}-\beta_{j, k}\right| \\
& =\left|\frac{1}{n} \sum_{v=1}^{n}\left(Q_{v} \mathbf{1}_{\left\{\left|Q_{v}\right| \leq \eta_{j}\right\}}-\mathbb{E}\left(Q_{v} \mathbf{1}_{\left\{\left|Q_{v}\right| \leq \eta_{j}\right\}}\right)\right)-\frac{1}{n} \sum_{v=1}^{n} \mathbb{E}\left(Q_{v} \mathbf{1}_{\left\{\left|Q_{v}\right|>\eta_{j}\right\}}\right)\right| \\
& \leq\left|\frac{1}{n} \sum_{v=1}^{n}\left(Q_{v} \mathbf{1}_{\left\{\left|Q_{v}\right| \leq \eta_{j}\right\}}-\mathbb{E}\left(Q_{v} \mathbf{1}_{\left\{\left|Q_{v}\right| \leq \eta_{j}\right\}}\right)\right)\right|+\frac{1}{n} \sum_{v=1}^{n} \mathbb{E}\left(\left|Q_{v}\right| \mathbf{1}_{\left\{\left|Q_{v}\right|>\eta_{j}\right\}}\right) .
\end{aligned}
$$

By (32) we obtain

$$
\frac{1}{n} \sum_{v=1}^{n} \mathbb{E}\left(\left|Q_{v}\right| \mathbf{1}_{\left\{\left|Q_{v}\right|>\eta_{j}\right\}}\right) \leq \theta 2^{(\delta+q) j} \sqrt{\frac{\rho_{n} \ln \left(n / \rho_{n}\right)}{n}}=\lambda_{j} .
$$

Hence

$$
\begin{aligned}
& \mathbb{P}\left(\left|\widehat{\beta}_{j, k}-\beta_{j, k}\right| \geq \kappa \lambda_{j} / 2\right) \\
& \leq \mathbb{P}\left(\left|\frac{1}{n} \sum_{v=1}^{n}\left(Q_{v} \mathbf{1}_{\left\{\left|Q_{v}\right| \leq \eta_{j}\right\}}-\mathbb{E}\left(Q_{v} \mathbf{1}_{\left\{\left|Q_{v}\right| \leq \eta_{j}\right\}}\right)\right)\right| \geq(\kappa / 2-1) \lambda_{j}\right)
\end{aligned}
$$

Let us now present the Bernstein inequality (see Petrov (1995)).

Lemma 2 (Bernstein's inequality) Let $n \in \mathbb{N}^{*}$ and $\left(U_{v}\right)_{v \in\{1, \ldots, n\}}$ be $n$ zero mean independent random variables such that there exists a constant $M>0$ satisfying, for any $v \in\{1, \ldots, n\},\left|U_{v}\right| \leq M<\infty$. Then, for any $\lambda>0$, we have

$$
\mathbb{P}\left(\left|\sum_{v=1}^{n} U_{v}\right| \geq \lambda\right) \leq 2 \exp \left(-\frac{\lambda^{2}}{2\left(\sum_{v=1}^{n} \mathbb{E}\left(U_{v}^{2}\right)+\frac{\lambda M}{3}\right)}\right) .
$$

Set, for any $v \in\{1, \ldots, n\}$,

$$
U_{v}=Q_{v} \mathbf{1}_{\left\{\left|Q_{v}\right| \leq \eta_{j}\right\}}-\mathbb{E}\left(Q_{v} \mathbf{1}_{\left\{\left|Q_{v}\right| \leq \eta_{j}\right\}}\right) .
$$

Then, for any $v \in\{1, \ldots, n\}$, we have $\mathbb{E}\left(U_{v}\right)=0$,

$$
\left|U_{v}\right| \leq\left|Q_{v}\right| \mathbf{1}_{\left\{\left|Q_{v}\right| \leq \eta_{j}\right\}}+\mathbb{E}\left(\left|Q_{v}\right| \mathbf{1}_{\left\{\left|Q_{v}\right| \leq \eta_{j}\right\}}\right) \leq 2 \eta_{j}
$$


and, using again Proposition 2 with $\psi$ instead of $\phi$,

$$
\begin{aligned}
\sum_{v=1}^{n} \mathbb{E}\left(\left|U_{v}\right|^{2}\right) & =\sum_{v=1}^{n} \mathbb{V}\left(Q_{v} \mathbf{1}_{\left\{\left|Q_{v}\right| \leq \eta_{j}\right\}}\right) \leq \sum_{v=1}^{n} \mathbb{E}\left(\left|Q_{v}\right|^{2}\right) \leq \theta^{2} 2^{2(\delta+q) j} \sum_{v=1}^{n} a_{\omega}^{2}(v) \\
& \leq \theta^{2} 2^{2(\delta+q) j} n \rho_{n} .
\end{aligned}
$$

It follows from the Bernstein inequality that

$$
\begin{aligned}
& \mathbb{P}\left(\left|\sum_{v=1}^{n} U_{v}\right| \geq n(\kappa / 2-1) \lambda_{n}\right) \\
& \leq 2 \exp \left(-\frac{n^{2}(\kappa / 2-1)^{2} \lambda_{j}^{2}}{2\left(\theta^{2} 2^{2(\delta+q) j} n \rho_{n}+\frac{2 n(\kappa / 2-1) \lambda_{j} \eta_{j}}{3}\right)}\right) .
\end{aligned}
$$

Remark that

$$
\lambda_{j} \eta_{j}=\theta 2^{(\delta+q) j} \sqrt{\frac{\rho_{n} \ln \left(n / \rho_{n}\right)}{n}} \theta 2^{(\delta+q) j} \sqrt{\frac{n \rho_{n}}{\ln \left(n / \rho_{n}\right)}}=\theta^{2} 2^{2(\delta+q) j} \rho_{n}
$$

and

$$
\lambda_{j}^{2}=\theta^{2} 2^{2(\delta+q) j} \frac{\rho_{n} \ln \left(n / \rho_{n}\right)}{n} .
$$

Combining (34) and (35), for any $\kappa \geq 8 / 3+2+2 \sqrt{16 / 9+4}$, we obtain

$$
\begin{aligned}
\mathbb{P}\left(\left|\widehat{\beta}_{j, k}-\beta_{j, k}\right| \geq \kappa \lambda_{j} / 2\right) & \leq 2 \exp \left(-\frac{(\kappa / 2-1)^{2} \ln \left(n / \rho_{n}\right)}{2\left(1+\frac{2(\kappa / 2-1)}{3}\right)}\right) \\
& =2\left(\frac{n}{\rho_{n}}\right)^{-\frac{(\kappa / 2-1)^{2}}{2\left(1+\frac{(\kappa / 2-1)}{3}\right)}} \leq 2\left(\frac{\rho_{n}}{n}\right)^{2} .
\end{aligned}
$$

This completes the proof of Proposition 5.

6.2 Proofs of the main results

Proof of Theorem 1. We expand the function $f_{\omega}^{(q)}$ on $\mathcal{B}$ as

$$
f_{\omega}^{(q)}(x)=\sum_{k=0}^{2^{j_{0}}-1} \alpha_{j_{0}, k} \phi_{j_{0}, k}(x)+\sum_{j=j_{0}}^{\infty} \sum_{k=0}^{2^{j}-1} \beta_{j, k} \psi_{j, k}(x),
$$

where

$$
\alpha_{j_{0}, k}=\int_{0}^{1} f_{\omega}^{(q)}(x) \bar{\phi}_{j_{0}, k}(x) d x, \quad \beta_{j, k}=\int_{0}^{1} f_{\omega}^{(q)}(x) \bar{\psi}_{j, k}(x) d x .
$$


We have

$$
\widehat{f}_{L}^{(q)}(x)-f_{\omega}^{(q)}(x)=\sum_{k=0}^{2^{j_{0}}-1}\left(\widehat{\alpha}_{j_{0}, k}-\alpha_{j_{0}, k}\right) \phi_{j_{0}, k}(x)-\sum_{j=j_{0}}^{\infty} \sum_{k=0}^{2^{j}-1} \beta_{j, k} \psi_{j, k}(x) .
$$

Since $\mathcal{B}$ is an orthonormal basis of $\mathbb{L}_{\text {per }}^{2}([0,1])$, we have

$$
\mathbb{E}\left(\int_{0}^{1}\left(\widehat{f}_{L}^{(q)}(x)-f_{\omega}^{(q)}(x)\right)^{2} d x\right)=A+B,
$$

where

$$
A=\sum_{k=0}^{2^{j_{0}}-1} \mathbb{E}\left(\left|\widehat{\alpha}_{j_{0}, k}-\alpha_{j_{0}, k}\right|^{2}\right), \quad B=\sum_{j=j_{0}}^{\infty} \sum_{k=0}^{2^{j}-1}\left|\beta_{j, k}\right|^{2} .
$$

Using Proposition 3, we obtain

$$
A \leq C 2^{j_{0}(1+2 \delta+2 q)} \frac{\rho_{n}}{n} \leq C\left(\frac{\rho_{n}}{n}\right)^{2 s /(2 s+2 \delta+2 q+1)} .
$$

Since $p \geq 2$, we have $B_{p, r}^{s}(M) \subseteq B_{2, \infty}^{s}(M)$. Hence

$$
B \leq C 2^{-2 j_{0} s} \leq C\left(\frac{\rho_{n}}{n}\right)^{2 s /(2 s+2 \delta+2 q+1)} .
$$

Therefore

$$
\mathbb{E}\left(\int_{0}^{1}\left(\widehat{f}_{L}^{(q)}(x)-f_{\omega}^{(q)}(x)\right)^{2} d x\right) \leq C\left(\frac{\rho_{n}}{n}\right)^{2 s /(2 s+2 \delta+2 q+1)} .
$$

The proof of Theorem 1 is complete.

Proof of Theorem 2. We expand the function $f_{\omega}^{(q)}$ on $\mathcal{B}$ as

$$
f_{\omega}^{(q)}(x)=\sum_{k=0}^{2^{j_{*}}-1} \alpha_{j_{*}, k} \phi_{j_{*}, k}(x)+\sum_{j=j_{*}}^{\infty} \sum_{k=0}^{2^{j}-1} \beta_{j, k} \psi_{j, k}(x)
$$

where

$$
\alpha_{j_{*}, k}=\int_{0}^{1} f_{\omega}^{(q)}(x) \bar{\phi}_{j_{*}, k}(x) d x, \quad \beta_{j, k}=\int_{0}^{1} f_{\omega}^{(q)}(x) \bar{\psi}_{j, k}(x) d x .
$$

We have

$$
\begin{aligned}
& \hat{f}_{H}^{(q)}(x)-f_{\omega}^{(q)}(x) \\
& =\sum_{k=0}^{2^{j *}-1}\left(\widehat{\alpha}_{j_{*}, k}-\alpha_{j_{*}, k}\right) \phi_{j_{*}, k}(x)+\sum_{j=j_{*}}^{j_{1}} \sum_{k=0}^{2^{j}-1}\left(\widehat{\beta}_{j, k} \mathbf{1}_{\left\{\left|\widehat{\beta}_{j, k}\right| \geq \kappa \lambda_{j}\right\}}-\beta_{j, k}\right) \psi_{j, k}(x) \\
& -\sum_{j=j_{1}+1}^{\infty} \sum_{k=0}^{2^{j}-1} \beta_{j, k} \psi_{j, k}(x) .
\end{aligned}
$$


Since $\mathcal{B}$ is an orthonormal basis of $\mathbb{L}_{\text {per }}^{2}([0,1])$, we have

$$
\mathbb{E}\left(\int_{0}^{1}\left(\widehat{f}_{H}^{(q)}(x)-f_{\omega}^{(q)}(x)\right)^{2} d x\right)=R+S+T
$$

where

$R=\sum_{k=0}^{2^{j *}-1} \mathbb{E}\left(\left|\widehat{\alpha}_{j_{*}, k}-\alpha_{j_{*}, k}\right|^{2}\right), \quad S=\sum_{j=j_{*}}^{j_{1}} \sum_{k=0}^{2^{j}-1} \mathbb{E}\left(\left|\widehat{\beta}_{j, k} \mathbf{1}_{\left\{\left|\widehat{\beta}_{j, k}\right| \geq \kappa \lambda_{j}\right\}}-\beta_{j, k}\right|^{2}\right)$

and

$$
T=\sum_{j=j_{1}+1}^{\infty} \sum_{k=0}^{2^{j}-1}\left|\beta_{j, k}\right|^{2}
$$

Let us bound $R, T$ and $S$ in turn.

Using Proposition 3 and the inequalities: $\rho_{n}<n / e, \rho_{n} \ln \left(n / \rho_{n}\right)<n$ and $2 s /(2 s+2 \delta+2 q+1)<1$, we obtain

$$
R \leq C 2^{j_{*}(1+2 \delta+2 q)} \frac{\rho_{n}}{n} \leq C \frac{\rho_{n}}{n} \leq C\left(\frac{\rho_{n} \ln \left(n / \rho_{n}\right)}{n}\right)^{2 s /(2 s+2 \delta+2 q+1)} .
$$

For $r \geq 1$ and $p \geq 2$, we have $B_{p, r}^{s}(M) \subseteq B_{2, \infty}^{s}(M)$. Since $\rho_{n} \ln \left(n / \rho_{n}\right)<n$ and $2 s /(2 s+2 \delta+2 q+1)<2 s /(2 \delta+2 q+1)$, we have

$$
\begin{aligned}
T & \leq C \sum_{j=j_{1}+1}^{\infty} 2^{-2 j s} \leq C 2^{-2 j_{1} s} \leq C\left(\frac{\rho_{n} \ln \left(n / \rho_{n}\right)}{n}\right)^{2 s /(2 \delta+2 q+1)} \\
& \leq C\left(\frac{\rho_{n} \ln \left(n / \rho_{n}\right)}{n}\right)^{2 s /(2 s+2 \delta+2 q+1)} .
\end{aligned}
$$

For $r \geq 1$ and $p \in[1,2)$, we have $B_{p, r}^{s}(M) \subseteq B_{2, \infty}^{s+1 / 2-1 / p}(M)$. Since $s>$ $(2 \delta+2 q+1) / p$, we have $(s+1 / 2-1 / p) /(2 \delta+2 q+1)>s /(2 s+2 \delta+2 q+1)$. So, using again $\rho_{n} \ln \left(n / \rho_{n}\right)<n$,

$$
\begin{aligned}
T & \leq C \sum_{j=j_{1}+1}^{\infty} 2^{-2 j(s+1 / 2-1 / p)} \leq C 2^{-2 j_{1}(s+1 / 2-1 / p)} \\
& \leq C\left(\frac{\rho_{n} \ln \left(n / \rho_{n}\right)}{n}\right)^{2(s+1 / 2-1 / p) /(2 \delta+2 q+1)} \leq C\left(\frac{\rho_{n} \ln \left(n / \rho_{n}\right)}{n}\right)^{2 s /(2 s+2 \delta+2 q+1)} .
\end{aligned}
$$

Hence, for $r \geq 1,\{p \geq 2$ and $s>0\}$ or $\{p \in[1,2)$ and $s>(2 \delta+2 q+1) / p\}$, we have

$$
T \leq C\left(\frac{\rho_{n} \ln \left(n / \rho_{n}\right)}{n}\right)^{2 s /(2 s+2 \delta+2 q+1)}
$$


The term $S$ can be decomposed as

$$
S=S_{1}+S_{2}+S_{3}+S_{4}
$$

where

$$
\begin{gathered}
S_{1}=\sum_{j=j_{*}}^{j_{1}} \sum_{k=0}^{2^{j}-1} \mathbb{E}\left(\left|\widehat{\beta}_{j, k}-\beta_{j, k}\right|^{2} \mathbf{1}_{\left\{\left|\widehat{\beta}_{j, k}\right| \geq \kappa \lambda_{j}\right\}} \mathbf{1}_{\left\{\left|\beta_{j, k}\right|<\kappa \lambda_{j} / 2\right\}}\right) \\
S_{2}=\sum_{j=j_{*}}^{j_{1}} \sum_{k=0}^{2^{j}-1} \mathbb{E}\left(\left|\widehat{\beta}_{j, k}-\beta_{j, k}\right|^{2} \mathbf{1}_{\left\{\left|\widehat{\beta}_{j, k}\right| \geq \kappa \lambda_{j}\right\}} \mathbf{1}_{\left\{\left|\beta_{j, k}\right| \geq \kappa \lambda_{j} / 2\right\}}\right), \\
S_{3}=\sum_{j=j_{*}}^{j_{1}} \sum_{k=0}^{2^{j}-1} \mathbb{E}\left(\left|\beta_{j, k}\right|^{2} \mathbf{1}_{\left\{\left|\widehat{\beta}_{j, k}\right|<\kappa \lambda_{j}\right\}} \mathbf{1}_{\left\{\left|\beta_{j, k}\right| \geq 2 \kappa \lambda_{j}\right\}}\right)
\end{gathered}
$$

and

$$
S_{4}=\sum_{j=j_{*}}^{j_{1}} \sum_{k=0}^{2^{j}-1} \mathbb{E}\left(\left|\beta_{j, k}\right|^{2} \mathbf{1}_{\left\{\left|\widehat{\beta}_{j, k}\right|<\kappa \lambda_{j}\right\}} \mathbf{1}_{\left\{\left|\beta_{j, k}\right|<2 \kappa \lambda_{j}\right\}}\right) .
$$

Let us analyze each term $S_{1}, S_{2}, S_{3}$ and $S_{4}$ in turn.

Upper bounds for $S_{1}$ and $S_{3}$. We have

$$
\begin{aligned}
& \left\{\left|\widehat{\beta}_{j, k}\right|<\kappa \lambda_{j},\left|\beta_{j, k}\right| \geq 2 \kappa \lambda_{j}\right\} \subseteq\left\{\left|\widehat{\beta}_{j, k}-\beta_{j, k}\right|>\kappa \lambda_{j} / 2\right\}, \\
& \left\{\left|\widehat{\beta}_{j, k}\right| \geq \kappa \lambda_{j},\left|\beta_{j, k}\right|<\kappa \lambda_{j} / 2\right\} \subseteq\left\{\left|\widehat{\beta}_{j, k}-\beta_{j, k}\right|>\kappa \lambda_{j} / 2\right\}
\end{aligned}
$$

and

$$
\left\{\left|\widehat{\beta}_{j, k}\right|<\kappa \lambda_{j},\left|\beta_{j, k}\right| \geq 2 \kappa \lambda_{j}\right\} \subseteq\left\{\left|\beta_{j, k}\right| \leq 2\left|\widehat{\beta}_{j, k}-\beta_{j, k}\right|\right\}
$$

So

$$
\max \left(S_{1}, S_{3}\right) \leq C \sum_{j=j_{*}}^{j_{1}} \sum_{k=0}^{2^{j}-1} \mathbb{E}\left(\left|\widehat{\beta}_{j, k}-\beta_{j, k}\right|^{2} \mathbf{1}_{\left\{\left|\widehat{\beta}_{j, k}-\beta_{j, k}\right|>\kappa \lambda_{j} / 2\right\}}\right)
$$

It follows from the Cauchy-Schwarz inequality and Propositions 4 and 5 that

$$
\begin{aligned}
& \mathbb{E}\left(\left|\widehat{\beta}_{j, k}-\beta_{j, k}\right|^{2} \mathbf{1}_{\left\{\left|\widehat{\beta}_{j, k}-\beta_{j, k}\right|>\kappa \lambda_{j} / 2\right\}}\right) \\
& \leq\left(\mathbb{E}\left(\left|\widehat{\beta}_{j, k}-\beta_{j, k}\right|^{4}\right)\right)^{1 / 2}\left(\mathbb{P}\left(\left|\widehat{\beta}_{j, k}-\beta_{j, k}\right|>\kappa \lambda_{j} / 2\right)\right)^{1 / 2} \\
& \leq C 2^{2(\delta+q) j} \frac{\rho_{n}^{2} \ln \left(n / \rho_{n}\right)}{n^{2}} .
\end{aligned}
$$


Since $\rho_{n} \ln \left(n / \rho_{n}\right)<n$ and $2 s /(2 s+2 \delta+2 q+1)<1$, we have

$$
\begin{aligned}
\max \left(S_{1}, S_{3}\right) & \leq C \frac{\rho_{n}^{2} \ln \left(n / \rho_{n}\right)}{n^{2}} \sum_{j=j_{*}}^{j_{1}} 2^{j(1+2 \delta+2 q)} \leq C \frac{\rho_{n}^{2} \ln \left(n / \rho_{n}\right)}{n^{2}} 2^{j_{1}(1+2 \delta+2 q)} \\
& \leq C \frac{\rho_{n} \ln \left(n / \rho_{n}\right)}{n} \leq C\left(\frac{\rho_{n} \ln \left(n / \rho_{n}\right)}{n}\right)^{2 s /(2 s+2 \delta+2 q+1)}
\end{aligned}
$$

Upper bound for $S_{2}$. Using Proposition 4 and the Cauchy-Schwarz inequality, we obtain

$$
\mathbb{E}\left(\left|\widehat{\beta}_{j, k}-\beta_{j, k}\right|^{2}\right) \leq\left(\mathbb{E}\left(\left|\widehat{\beta}_{j, k}-\beta_{j, k}\right|^{4}\right)\right)^{1 / 2} \leq C 2^{2(\delta+q) j} \frac{\rho_{n} \ln \left(n / \rho_{n}\right)}{n} .
$$

Hence

$$
S_{2} \leq C \frac{\rho_{n} \ln \left(n / \rho_{n}\right)}{n} \sum_{j=j_{*}}^{j_{1}} 2^{2(\delta+q) j} \sum_{k=0}^{2^{j}-1} \mathbf{1}_{\left\{\left|\beta_{j, k}\right|>\kappa \lambda_{j} / 2\right\}} .
$$

Let $j_{2}$ be the integer defined by

$$
\frac{1}{2}\left(\frac{n}{\rho_{n} \ln \left(n / \rho_{n}\right)}\right)^{1 /(2 s+2 \delta+2 q+1)}<2^{j_{2}} \leq\left(\frac{n}{\rho_{n} \ln \left(n / \rho_{n}\right)}\right)^{1 /(2 s+2 \delta+2 q+1)}
$$

We have

$$
S_{2} \leq S_{2,1}+S_{2,2}
$$

where

$$
S_{2,1}=C \frac{\rho_{n} \ln \left(n / \rho_{n}\right)}{n} \sum_{j=j_{*}}^{j_{2}} 2^{2(\delta+q) j} \sum_{k=0}^{2^{j}-1} \mathbf{1}_{\left\{\left|\beta_{j, k}\right|>\kappa \lambda_{j} / 2\right\}}
$$

and

$$
S_{2,2}=C \frac{\rho_{n} \ln \left(n / \rho_{n}\right)}{n} \sum_{j=j_{2}+1}^{j_{1}} 2^{2(\delta+q) j} \sum_{k=0}^{2^{j}-1} \mathbf{1}_{\left\{\left|\beta_{j, k}\right|>\kappa \lambda_{j} / 2\right\}}
$$

We have

$$
\begin{aligned}
S_{2,1} & \leq C \frac{\rho_{n} \ln \left(n / \rho_{n}\right)}{n} \sum_{j=j_{*}}^{j_{2}} 2^{j(1+2 \delta+2 q)} \leq C \frac{\rho_{n} \ln \left(n / \rho_{n}\right)}{n} 2^{j_{2}(1+2 \delta+2 q)} \\
& \leq C\left(\frac{\rho_{n} \ln \left(n / \rho_{n}\right)}{n}\right)^{2 s /(2 s+2 \delta+2 q+1)} .
\end{aligned}
$$

For $r \geq 1$ and $p \geq 2$, since $B_{p, r}^{s}(M) \subseteq B_{2, \infty}^{s}(M)$,

$$
\begin{aligned}
S_{2,2} & \leq C \frac{\rho_{n} \ln \left(n / \rho_{n}\right)}{n} \sum_{j=j_{2}+1}^{j_{1}} 2^{2(\delta+q) j} \frac{1}{\lambda_{j}^{2}} \sum_{k=0}^{2^{j}-1}\left|\beta_{j, k}\right|^{2} \leq C \sum_{j=j_{2}+1}^{\infty} \sum_{k=0}^{2^{j}-1}\left|\beta_{j, k}\right|^{2} \\
& \leq C \sum_{j=j_{2}+1}^{\infty} 2^{-2 j s} \leq C 2^{-2 j_{2} s} \leq C\left(\frac{\rho_{n} \ln \left(n / \rho_{n}\right)}{n}\right)^{2 s /(2 s+2 \delta+2 q+1)} .
\end{aligned}
$$


For $r \geq 1, p \in[1,2)$ and $s>(2 \delta+2 q+1) / p$, since $B_{p, r}^{s}(M) \subseteq B_{2, \infty}^{s+1 / 2-1 / p}(M)$ and $(2 s+2 \delta+2 q+1)(2-p) / 2+(s+1 / 2-1 / p+\delta+q-2(\delta+q) / p) p=2 s$, we have

$$
\begin{aligned}
S_{2,2} & \leq C \frac{\rho_{n} \ln \left(n / \rho_{n}\right)}{n} \sum_{j=j_{2}+1}^{j_{1}} 2^{2(\delta+q) j} \frac{1}{\lambda_{j}^{p}} \sum_{k=0}^{2^{j}-1}\left|\beta_{j, k}\right|^{p} \\
& \leq C\left(\frac{\rho_{n} \ln \left(n / \rho_{n}\right)}{n}\right)^{(2-p) / 2} \sum_{j=j_{2}+1}^{\infty} 2^{j(\delta+q)(2-p)} 2^{-j(s+1 / 2-1 / p) p} \\
& \leq C\left(\frac{\rho_{n} \ln \left(n / \rho_{n}\right)}{n}\right)^{(2-p) / 2} 2^{-j_{2}(s+1 / 2-1 / p+\delta+q-2(\delta+q) / p) p} \\
& \leq C\left(\frac{\rho_{n} \ln \left(n / \rho_{n}\right)}{n}\right)^{2 s /(2 s+2 \delta+2 q+1)} .
\end{aligned}
$$

So, for $r \geq 1,\{p \geq 2$ and $s>0\}$ or $\{p \in[1,2)$ and $s>(2 \delta+2 q+1) / p\}$, we have

$$
S_{2} \leq C\left(\frac{\rho_{n} \ln \left(n / \rho_{n}\right)}{n}\right)^{2 s /(2 s+2 \delta+2 q+1)} .
$$

Upper bound for $S_{4}$. We have

$$
S_{4} \leq \sum_{j=j_{*}}^{j_{1}} \sum_{k=0}^{2^{j}-1}\left|\beta_{j, k}\right|^{2} \mathbf{1}_{\left\{\left|\beta_{j, k}\right|<2 \kappa \lambda_{j}\right\}}
$$

Let $j_{2}$ be the integer (41). We have

$$
S_{4} \leq S_{4,1}+S_{4,2},
$$

where

$S_{4,1}=\sum_{j=j_{*}}^{j_{2}} \sum_{k=0}^{2^{j}-1}\left|\beta_{j, k}\right|^{2} \mathbf{1}_{\left\{\left|\beta_{j, k}\right|<2 \kappa \lambda_{j}\right\}}, \quad S_{4,2}=\sum_{j=j_{2}+1}^{j_{1}} \sum_{k=0}^{2^{j}-1}\left|\beta_{j, k}\right|^{2} \mathbf{1}_{\left\{\left|\beta_{j, k}\right|<2 \kappa \lambda_{j}\right\}}$.

We have

$$
\begin{aligned}
S_{4,1} & \leq C \sum_{j=j_{*}}^{j_{2}} 2^{j} \lambda_{j}^{2}=C \frac{\rho_{n} \ln \left(n / \rho_{n}\right)}{n} \sum_{j=j_{*}}^{j_{2}} 2^{j(1+2 \delta+2 q)} \leq C \frac{\rho_{n} \ln \left(n / \rho_{n}\right)}{n} 2^{j_{2}(1+2 \delta+2 q)} \\
& \leq C\left(\frac{\rho_{n} \ln \left(n / \rho_{n}\right)}{n}\right)^{2 s /(2 s+2 \delta+2 q+1)} .
\end{aligned}
$$

For $r \geq 1$ and $p \geq 2$, since $B_{p, r}^{s}(M) \subseteq B_{2, \infty}^{s}(M)$, we have

$$
\begin{aligned}
S_{4,2} & \leq \sum_{j=j_{2}+1}^{\infty} \sum_{k=0}^{2^{j}-1}\left|\beta_{j, k}\right|^{2} \leq C \sum_{j=j_{2}+1}^{\infty} 2^{-2 j s} \leq C 2^{-2 j_{2} s} \\
& \leq C\left(\frac{\rho_{n} \ln \left(n / \rho_{n}\right)}{n}\right)^{2 s /(2 s+2 \delta+2 q+1)} .
\end{aligned}
$$


For $r \geq 1, p \in[1,2)$ and $s>(2 \delta+2 q+1) / p$, since $B_{p, r}^{s}(M) \subseteq B_{2, \infty}^{s+1 / 2-1 / p}(M)$ and $(2 s+2 \delta+2 q+1)(2-p) / 2+(s+1 / 2-1 / p+\delta+q-2(\delta+q) / p) p=2 s$, we have

$$
\begin{aligned}
S_{4,2} & \leq C \sum_{j=j_{2}+1}^{j_{1}} \lambda_{j}^{2-p} \sum_{k=0}^{2^{j}-1}\left|\beta_{j, k}\right|^{p} \\
& =C\left(\frac{\rho_{n} \ln \left(n / \rho_{n}\right)}{n}\right)^{(2-p) / 2} \sum_{j=j_{2}+1}^{j_{1}} 2^{j(\delta+q)(2-p)} \sum_{k=0}^{2^{j}-1}\left|\beta_{j, k}\right|^{p} \\
& \leq C\left(\frac{\rho_{n} \ln \left(n / \rho_{n}\right)}{n}\right)^{(2-p) / 2} \sum_{j=j_{2}+1}^{\infty} 2^{j(\delta+q)(2-p)} 2^{-j(s+1 / 2-1 / p) p} \\
& \leq C\left(\frac{\rho_{n} \ln \left(n / \rho_{n}\right)}{n}\right)^{(2-p) / 2} 2^{-j_{2}(s+1 / 2-1 / p+\delta+q-2(\delta+q) / p) p} \\
& \leq C\left(\frac{\rho_{n} \ln \left(n / \rho_{n}\right)}{n}\right)^{2 s /(2 s+2 \delta+2 q+1)} .
\end{aligned}
$$

So, for $r \geq 1,\{p \geq 2$ and $s>0\}$ or $\{p \in[1,2)$ and $s>(2 \delta+2 q+1) / p\}$, we have

$$
S_{4} \leq C\left(\frac{\rho_{n} \ln \left(n / \rho_{n}\right)}{n}\right)^{2 s /(2 s+2 \delta+2 q+1)} .
$$

It follows from (39), (40), (42) and (43) that

$$
S \leq C\left(\frac{\rho_{n} \ln \left(n / \rho_{n}\right)}{n}\right)^{2 s /(2 s+2 \delta+2 q+1)}
$$

Combining (36), (37), (38) and (44), we have, for $r \geq 1,\{p \geq 2$ and $s>0\}$ or $\{p \in[1,2)$ and $s>(2 \delta+2 q+1) / p\}$,

$$
\mathbb{E}\left(\int_{0}^{1}\left(\widehat{f}_{H}^{(q)}(x)-f_{\omega}^{(q)}(x)\right)^{2} d x\right) \leq C\left(\frac{\rho_{n} \ln \left(n / \rho_{n}\right)}{n}\right)^{2 s /(2 s+2 \delta+2 q+1)} .
$$

The proof of Theorem 2 is complete.

Acknowledgments. The author thanks two anonymous referees for their thorough and useful comments. This work is supported by ANR grant NatImages, ANR-08-EMER-009. 


\section{References}

Bhattacharya, P.K., 1967. Estimation of a probability density function and its derivatives, Sankhya, Serie A, 29, 373-382.

Cai, T., 1999. Adaptive Wavelet Estimation: A Block Thresholding And Oracle Inequality Approach. The Annals of Statistics, 27, 898-924.

Cai, T., 2002. On adaptive wavelet estimation of a derivative and other related linear inverse problems. Journal of Statistical Planning and Inference, 108, 329-349.

Cavalier, L., Tsybakov, A.B. 2002. Sharp adaptation for inverse problems with random noise, Probability Theory and Related Fields, 123, 3,323-354.

Cavalier, L., Raimondo, M. 2007. Wavelet deconvolution with noisy eigenvalues. IEEE Transactions on signal processing, 55, 2414-2424.

Chaubey, Y.P., Doosti, H., 2005. Wavelet based estimation of the derivatives of a density for $m$-dependent random variables, Journal of the Iranian Statistical Society, 4, (2) , 97-105.

Chaubey, Y.P., Doosti, H., Prakasa Rao, B.L.S., 2006. Wavelet based estimation of the derivatives of a density with associated variables, International Journal of Pure and Applied Mathematics, 27, (1), 97-106.

Chaubey, Y.P., Doosti, H., Prakasa Rao, B.L.S., 2008. Wavelet based estimation of the derivatives of a density for a negatively associated process, Journal of Statistical Theory and Practice, 2, (3), 453-463.

Chesneau, C., 2008. Wavelet estimation via block thresholding: A minimax study under the $\mathbb{L}^{p}$ risk, Statistica Sinica, 18, 3, 1007-1024.

Chesneau, C., 2010. Wavelet estimation of the derivatives of an unknown function from a convolution model, Current Development in Theory and Applications of Wavelets (to appear).

Cohen, A., Daubechies, I., Jawerth, B., Vial, P., 1993. Wavelets on the interval and fast wavelet transforms. Applied and Computational Harmonic Analysis, 24, 1, 54-81.

Delyon, B., Juditsky, A., 1996. On minimax wavelet estimators. Applied Computational Harmonic Analysis, 3, 215-228.

Donoho, D. L., Raimondo, M., 2004. Translation invariant deconvolution in a periodic setting. The International Journal of Wavelets, Multiresolution and Information Processing, 2, (4):415-432.

Fan, J., Koo, J.Y., 2002. Wavelet deconvolution. IEEE transactions on information theory, $48,734-747$.

Johnstone, I., Kerkyacharian, G., Picard, D., Raimondo, M., 2004. Wavelet deconvolution in a periodic setting. Journal of the Royal Statistical Society, Serie B, 66 (3), 547-573.

Kerkyacharian, G., Picard, D., Raimondo, M., 2007. Adaptive boxcar deconvolution on full Lebesgue measure sets. Statistica Sinica, 17, 317-340.

Maiboroda, R. E., 1996. Estimators of components of a mixture with varying concentrations. Ukrain. Mat. Zh., 48, 4, 562-566. 
Meyer, Y., 1992. Wavelets and Operators. Cambridge University Press, Cambridge.

Pensky, M., Vidakovic, B., 1999. Adaptive wavelet estimator for nonparametric density deconvolution. The Annals of Statistics, 27, 2033-2053.

Pensky, M., Sapatinas, T., 2009. Functional deconvolution in a periodic setting: Uniform Case. The Annals of Statistics, 37, 1, 73-104.

Petrov, V.V., 1995. Limit Theorems of Probability Theory: Sequences of Independent Random Variables. Oxford: Clarendon Press.

Petsa, A., Sapatinas, T., 2009. Minimax convergence rates under the $\mathbb{L}^{p}$-risk in the functional deconvolution model. Statistics and Probability Letters, 79, 1568-1576.

Pokhyl'ko, D., 2005. Wavelet estimators of a density constructed from observations of a mixture. Theory of Probability and Mathematical Statististics, 70, 135-145.

Prakasa Rao, B.L.S., 1996. Nonparametric estimation of the derivatives of a density by the method of wavelets, Bull. Inform. Cyb., 28, 91-100.

Prakasa Rao, B.L.S., 2010. Wavelet linear estimation for derivatives of a density from observations of mixtures with varying mixing proportions. Indian Journal of Pure and Applied Mathematics, 41, 1, 275-291.

Rosenthal, H.P., 1970. On the subspaces of $\mathbb{L}^{p}(p \geq 2)$ spanned by sequences of independent random variables. Israel Journal of Mathematics, 8, 273-303.

Tsybakov, A., 2004. Introduction à l'estimation nonparamétrique, Springer Verlag, Berlin.

Willer, T., 2005. Deconvolution in white noise with a random blurring effect. Preprint LPMA. 